RUB-TP2-19/00

TPR-00-15

\title{
DVCS on the nucleon to the twist-3 accuracy
}

\author{
N. Kivel ${ }^{a, b}$, M.V. Polyakov ${ }^{b, c}$ \\ ${ }^{a}$ Institut für Theoretische Physik, Universität Regensburg \\ D-93040 Regensburg, Germany \\ ${ }^{b}$ Petersburg Nuclear Physics Institute, 188350, Gatchina, Russia \\ ${ }^{c}$ Institut für Theoretische Physik II, Ruhr-Universität Bochum, \\ D-44780 Bochum, Germany
}

\begin{abstract}
The amplitude of the deeply virtual Compton scattering off nucleon is computed to the twist-3 accuracy in the Wandzura-Wilczek (WW) approximation. The result is presented in the form which can be easily used for analysis of DVCS observables.
\end{abstract}

\section{Introduction}

Deeply virtual Compton scattering (DVCS) [1, 2] is the cleanest hard process which is sensitive to the skewed parton distributions (SPD) and has been the subject of extensive theoretical investigations for a few years. First experimental data became recently available (see e.g. [3, 4, 5]) and much more data are expected from JLAB, DESY, and CERN in the near future. The leading order $\left(1 / Q^{0}\right)$ DVCS amplitude owing to factorisation theorems [6, 7, 8] can be expressed in terms of twist-2 skewed parton distributions. However, as the typical experimentally accessible $Q^{2}$ are by no means large, studies of the power (higher twist) corrections to the DVCS amplitude are very important. The leading power corrections to the DVCS amplitude are of the order $1 / Q$ only (hence twist-3) and their effect on the DVCS observables can be rather sizeable. Also the size of the twist-3 corrections increases with the increase of the momentum transfer squared $t$ because they enter the amplitude typically as $\sqrt{-t} / Q$. Therefore, taking into account the twist-3 corrections is important for the problem of continuation of the twist-2 SPD's to $t=0$.

Recently the DVCS amplitude was computed in Refs. [9, 10, 11, 12] including the terms of the order $O(1 / Q)$. The inclusion of such terms is mandatory to ensure the electromagnetic gauge invariance of the DVCS amplitude to the order $\sqrt{-t} / Q$. At the order $1 / Q$ the DVCS amplitude depends on a set of new skewed parton distributions. Recently it was shown in Ref. [11] that in the so-called Wandzura-Wilczek (WW) approximation these new functions can be expressed in terms of twist-2 SPD's.

The WW approximation corresponds to neglecting the nucleon matrix elements of the 'genuine twist-3' operators of the type $\bar{\psi} G \psi$, i.e. neglecting the nonperturbative quarkgluon correlations in the nucleon. In principle this approximation is not justified by any small parameter of the theory. However, recent measurements of the polarized structure function $g_{T}(x)$ 13 show that the WW approximation works pretty well in this case. Also the estimates of the 'genuine twist-3' contributions to polarized structure functions $g_{T}(x)$ and 
$h_{L}(x)$ in the theory of instanton vacuum [14, 15] (which is a model of non-perturbative quarkgluon correlations) showed that these contributions are parametrically suppressed relative to the 'kinematical' part of the twist-3 contributions by the packing fraction of instantons in the vacuum.

Generically the new twist-3 SPD's in the WW approximation posses discontinuities at the points $x= \pm \xi$ [12, 16] which potentially, may lead to divergencies in the DVCS amplitude and hence to the violation of the factorisation at the $1 / Q$ accuracy. However, it was shown in [12, 16] for the case of DVCS on the pion target that these dangerous divergencies are cancelled to the $1 / Q$ accuracy. In Ref. [16] general arguments were given that the cancellation of the divergencies should persist also for any target. In the present paper we demonstrate by explicit calculations that indeed the DVCS amplitude on the nucleon to the $1 / Q$ accuracy in the WW approximation is free of the divergencies. The results can easily be used for the analysis of the DVCS observables. Collection of formulae the reader can find in the Appendix.

\section{DVCS amplitude on the nucleon at the twist-3 accuracy}

We shall work in the reference frame where the average nucleon momenta and the virtual photon momentum are collinear and have opposite directions. For convenience we introduce light-cone vectors $n, n^{*}$ such that

$$
n \cdot n=0, n^{*} \cdot n^{*}=0, n \cdot n^{*}=1 .
$$

We also define the transverse metric and antisymmetric transverse epsilon tensor 冈:

$$
\left(-g^{\mu \nu}\right)_{\perp}=-g^{\mu \nu}+n^{\mu} n^{* \nu}+n^{\nu} n^{* \mu}, \quad \epsilon_{\mu \nu}^{\perp}=\epsilon_{\mu \nu \alpha \beta} n^{\alpha} n^{* \beta} .
$$

For an arbitrary vector $a_{\mu}$ we will use the shorthand notation

$$
a_{+} \equiv a_{\mu} n^{\mu}, \quad a_{-} \equiv a_{\mu} n^{* \nu}
$$

To the twist-3 accuracy the particle momenta can be written as:

$$
\begin{aligned}
& P=\frac{1}{2}\left(p+p^{\prime}\right)=n^{*}, \quad \Delta=p^{\prime}-p=-2 \xi P+\Delta_{\perp}, \\
& q=-2 \xi P+\frac{Q^{2}}{4 \xi} n, \quad q^{\prime}=q-\Delta=\frac{Q^{2}}{4 \xi} n-\Delta_{\perp},
\end{aligned}
$$

where $p, p^{\prime}$ are momenta of the initial and final nucleon and $q, q^{\prime}$ are momenta of the initial and final photon respectively.

The DVCS amplitude on the nucleon is related to the matrix element of T-product of two electromagnetic currents

$$
T^{\mu \nu}=-i \int d^{4} x e^{-i\left(q+q^{\prime}\right) x / 2}\left\langle p^{\prime}\left|T J^{\mu}(x / 2) J^{\nu}(-x / 2)\right| p\right\rangle .
$$

*The Levi-Civita tensor $\epsilon_{\mu \nu \alpha \beta}$ is defined as the totally antisymmetric tensor with $\epsilon_{0123}=1$ 
To the twist-3 accuracy it has been computed in Refs. [10, 11]. We use here the form of the amplitude obtained in [10] in framework of the parton model i]. This form is equivalent to that of Ref. [11], and can be written as:

$$
\begin{aligned}
T^{\mu \nu} & =\frac{1}{2} \int_{-1}^{1} d x\left\{\left[\left(-g^{\mu \nu}\right)_{\perp}-\frac{P^{\nu} \Delta_{\perp}^{\mu}}{(P q)}\right] n^{\rho} F_{\rho}(x, \xi) C^{+}(x, \xi)\right. \\
& -\left[\left(-g^{\nu k}\right)_{\perp}-\frac{P^{\nu} \Delta_{\perp}^{k}}{(P q)}\right] i \epsilon_{k \mu}^{\perp} n^{\rho} \widetilde{F}_{\rho}(x, \xi) C^{-}(x, \xi) \\
& -\frac{(q+4 \xi P)^{\mu}}{(P q)}\left[\left(-g^{\nu k}\right)_{\perp}-\frac{P^{\nu} \Delta_{\perp}^{k}}{(P q)}\right]\left\{F_{k}(x, \xi) C^{+}(x, \xi)-i \epsilon_{k \rho}^{\perp} \widetilde{F}_{\rho}(x, \xi) C^{-}(x, \xi)\right\} \\
& \left.+\frac{(q+2 \xi P)^{\nu}}{(P q)}\left\{F_{\mu_{\perp}}(x, \xi) C^{+}(x, \xi)+i \epsilon_{\mu \rho}^{\perp} \widetilde{F}_{\rho}(x, \xi) C^{-}(x, \xi)\right\}\right\},
\end{aligned}
$$

where the leading order coefficient functions are

$$
C^{ \pm}(x, \xi)=\frac{1}{x-\xi+i \varepsilon} \pm \frac{1}{x+\xi-i \varepsilon}
$$

and skewed distributions $F_{\mu}(x, \xi)$ and $\widetilde{F}_{\mu}(x, \xi)$ are defined in terms of the nonlocal light-cone quark operators f:

$$
\begin{aligned}
& F_{\mu}(x, \xi)=\int_{-\infty}^{\infty} \frac{d \lambda}{2 \pi} e^{-i x \lambda}\left\langle p^{\prime}\left|\bar{\psi}\left(\frac{1}{2} \lambda n\right) \gamma_{\mu} \psi\left(-\frac{1}{2} \lambda n\right)\right| p\right\rangle \\
& \widetilde{F}_{\mu}(x, \xi)=\int_{-\infty}^{\infty} \frac{d \lambda}{2 \pi} e^{-i x \lambda}\left\langle p^{\prime}\left|\bar{\psi}\left(\frac{1}{2} \lambda n\right) \gamma_{\mu} \gamma_{5} \psi\left(-\frac{1}{2} \lambda n\right)\right| p\right\rangle .
\end{aligned}
$$

We do not write the sum over quark flavours to simplify notations, the flavour dependence in Eqs. (可) can be restored easily by the substitution:

$$
\bar{\psi} \ldots \psi \rightarrow \sum_{f=u, d, s, \ldots} e_{f}^{2} \bar{\psi}_{f} \ldots \psi_{f}
$$

The amplitude (6) is electromagnetically gauge invariant:

$$
q_{\mu} T^{\mu \nu}=(q-\Delta)_{\nu} T^{\mu \nu}=0
$$

to the accuracy $1 / Q^{2}$. In order to have 'absolute' transversality of the amplitude we keep in the expression (6) terms of the $\Delta^{2} / Q^{2}$ order, applying the prescription of [17, 18] $\left(-g_{\perp}^{\mu \nu} \rightarrow\right.$ $\left.-g_{\perp}^{\mu \nu}-\frac{P^{\mu} \Delta_{\perp}^{\nu}}{(P q)}\right)$ for the twist-3 terms in the amplitude. Formally such terms are beyond our accuracy and they do not form a complete set of $1 / Q^{2}$ contributions, but we prefer to work with the transverse DVCS amplitude.

\footnotetext{
†We change little bit notations of Ref. 10

‡The gauge link between points on the light-cone is not shown but always assumed.
} 
It is easy to see that the third term in Eq. (6) corresponds to the contribution of the longitudinal polarisation of the virtual photon. Note that the last term in the expression (6), corresponding to transverse polarisation of the virtual photon, is proportional to $(q+2 \xi P)=$ $q^{\prime}+\Delta_{\perp}$. Hence, contracting with the transverse polarisation vector $e_{\nu}\left(q^{\prime}\right)$ of the final real photon we obtain

$$
e_{\nu}\left(q^{\prime}\right)(q+2 \xi P)^{\nu}=e_{\nu}\left(q^{\prime}\right) \Delta_{\perp}^{\nu}
$$

Therefore, such term does not contribute to any observable with the accuracy $O(\Delta / Q)$. The same contribution has been obtained for the DVCS on the pion. For the case of the pion target in Ref. [12] it was shown by a direct calculation that the structure $(q+2 \xi P)$ is the truncated form of the vector $q^{\prime}$ and hence such term has zero projection onto the polarisation vector of the final photon.

In what follows we compute the skewed parton distributions (7) in the so-called WandzuraWilczek (WW) approximation. This means that we neglect the contributions of the quarkgluon operators, which define what can be called 'genuine twist-3' part. We will demonstrate that at least in this approximation the amplitude possesses the factorisation property. Another point, assuming that the contribution of the quark-gluon correlations us smaller than the WW part, one can estimate the effects of the $1 / Q$ suppressed terms to various DVCS observables.

\section{Calculation of the matrix elements in the WW approximation}

The aim of this section is the calculation of the matrix elements (7) in the WW-approximation. To obtain the answer we make use of the operator identities derived in [11, 12, 16] on the basis of technique developed in ref. [19]:

$$
\begin{aligned}
\bar{\psi}(x) \gamma_{\mu} \psi(-x) & =\frac{1}{2} \int_{0}^{1} d \alpha\left\{\alpha \mathcal{D}_{\mu}\left(e^{-\bar{\alpha}(x \mathcal{D})}-e^{\bar{\alpha}(x \mathcal{D})}\right)+\left(e^{-\bar{\alpha}(x \mathcal{D})}+e^{\bar{\alpha}(x \mathcal{D})}\right) \frac{\partial}{\partial x_{\mu}}\right\} \bar{\psi}(\alpha x) \not \psi(-\alpha x) \\
& -i \epsilon_{\mu i j k} x^{i} \mathcal{D}^{j} \int_{0}^{1} d u e^{(2 u-1)(x \mathcal{D})} \int_{0}^{u} d \alpha e^{-\alpha(x \mathcal{D})} \frac{\partial}{\partial x_{k}} \bar{\psi}(\alpha x) \not \gamma \gamma_{5} \psi(-\alpha x)+\ldots,
\end{aligned}
$$

where $\bar{\alpha}=1-\alpha$ and ellipses stand for the contributions of either twist-3 quark gluon operators or twist- 4 operators 1$]$ which we neglect in the WW approximation. An analogous expression for the operator $\bar{\psi}(x) \gamma_{\mu} \gamma_{5} \psi(-x)$ can be obtained from Eq. (11) by the replacement $\not x \rightarrow \not x \gamma_{5}$ in RHS of this equation. Keeping in mind this trivial replacement we shall present all derivations only for the vector operator, the generalisation to axial vector operators is trivial.

The symbol $\mathcal{D}$ denotes the derivative with respect to the total translation:

$$
\left.\mathcal{D}_{\alpha}\{\bar{\psi}(t x) \Gamma[t x,-t x] \psi(-t x)\} \equiv \frac{\partial}{\partial y^{\alpha}}\{\bar{\psi}(t x+y) \Gamma[t x+y,-t x+y] \psi(-t x+y)\}\right|_{y \rightarrow 0}
$$

\footnotetext{
${ }^{\S}$ For simple derivation see Ref. [16]

T In Ref. [16] more general relation is derived in which the twist-4 terms of the kind $x^{2} \Delta^{2}$ are summed up.
} 
with a generic Dirac matrix structure $\Gamma$ and $[x, y]=\operatorname{Pexp}\left[i g \int_{0}^{1} d t(x-y)_{\mu} A^{\mu}(t x+(1-t) y)\right]$. Note that in the matrix elements the total derivative can be easily converted to the momentum transfer:

$$
\left\langle p^{\prime}\left|\mathcal{D}_{\mu} \bar{\psi}(t x) \Gamma[t x,-t x] \psi(-t x)\right| p\right\rangle=i\left(p^{\prime}-p\right)_{\mu}\left\langle p^{\prime}|\bar{\psi}(t x) \Gamma[t x,-t x] \psi(-t x)| p\right\rangle
$$

and therefore in the matrix elements one can associate $\mathcal{D}$ with the momentum transfer $\Delta$. For what follows it is convenient to rewrite the identity (11) in the more symmetric form: 1

$$
\begin{aligned}
\bar{\psi}(x) \gamma_{\mu} \psi(-x) & =\frac{\mathcal{D}_{\mu}}{(\mathcal{D} x)} \bar{\psi}(x) \not \psi \psi(-x) \\
& +\frac{1}{2} \int_{0}^{1} d \alpha\left\{e^{\bar{\alpha}(x \mathcal{D})}+e^{-\bar{\alpha}(x \mathcal{D})}\right\}\left[\partial_{\mu}-\frac{\mathcal{D}_{\mu}}{(x \mathcal{D})}(x \partial)\right] \bar{\psi}(\alpha x) \not x \psi(-\alpha x) \\
& +\frac{1}{2} \int_{0}^{1} d \alpha\left\{e^{-\bar{\alpha}(x \mathcal{D})}-e^{\bar{\alpha}(x \mathcal{D})}\right\} i \epsilon_{\mu i j k} x_{i} \frac{\mathcal{D}_{j}}{(\mathcal{D} x)} \partial_{k} \bar{\psi}(\alpha x) \not \gamma \gamma_{5} \psi(-\alpha x)+\ldots
\end{aligned}
$$

To obtain this expression one has to perform an integration by parts in the first and last terms of Eq. (11).

Our aim is to calculate the matrix elements $\left\langle p^{\prime}\left|\bar{\psi}(z) \gamma^{\mu}\left(\gamma_{5}\right) \psi(-z)\right| p\right\rangle$ in terms of the twist2 symmetrical matrix elements $\left\langle p^{\prime}\left|\bar{\psi}(x) \not x\left(\gamma_{5}\right) \psi(-x)\right| p\right\rangle$ which appear in the RHS of Eq. (14). At the first step, the symmetric matrix elements should be parametrised for arbitrary vector $x$ which is not light-like. The parametrisations of the twist- 2 matrix elements can be written as follows:

$$
\left\langle p^{\prime}|\bar{\psi}(x) \not \psi \psi(-x)| p\right\rangle=\langle\langle\not x\rangle\rangle T_{1}[(P x), \xi(x)]+\left\langle\left\langle\frac{i \sigma^{\alpha \beta} x_{\alpha} \Delta_{\beta}}{2 M}\right\rangle\right\rangle T_{2}[(P x), \xi(x)],
$$

where we use shorthand notations for:

$$
\xi(x)=-\frac{1}{2} \frac{(\Delta x)}{(P x)}, \quad\langle\langle\Gamma\rangle\rangle \equiv \bar{U}\left(p^{\prime}, S^{\prime}\right) \Gamma U(p, S),
$$

where $U(p, S)$ and $\bar{U}\left(p^{\prime}, S^{\prime}\right)$ denote initial and final nucleon spinors, respectively. The amplitudes $T_{1,2}[(P x), \xi(x)]$ can be parametrised in terms of the twist-2 skewed parton distribution $H, E$ of Ref. [2]:

$$
\begin{aligned}
& T_{1}[(P z), \xi]=\int_{-1}^{1} d u e^{i u 2(P z)} H(u, \xi), \\
& T_{2}[(P z), \xi]=\int_{-1}^{1} d u e^{i u 2(P z)} E(u, \xi) .
\end{aligned}
$$

Similar formulae can be written for the axial operator:

$$
\left\langle p^{\prime}\left|\bar{\psi}(x) \not \gamma_{5} \psi(-x)\right| p\right\rangle=\left\langle\left\langle\not \gamma_{5}\right\rangle\right\rangle \widetilde{T}_{1}[(P x), \xi(x)]+\left\langle\left\langle\frac{\gamma_{5}(\Delta x)}{2 M}\right\rangle \widetilde{T}_{2}[(P x), \xi(x)],\right.
$$

\footnotetext{
" for simplicity we use notation $\partial / \partial x_{\mu} \equiv \partial_{\mu}$
} 
with amplitudes $\widetilde{T}_{1,2}$ given in terms of twist-2 SPD's $\widetilde{H}, \widetilde{E}$ as:

$$
\begin{aligned}
& \widetilde{T}_{1}[(P z), \xi]=\int_{-1}^{1} d u e^{i u 2(P z)} \widetilde{H}(u, \xi), \\
& \widetilde{T}_{2}[(P z), \xi]=\int_{-1}^{1} d u e^{i u 2(P z)} \widetilde{E}(u, \xi) .
\end{aligned}
$$

Now, we have to insert the parametrisations (17) and (19) into the RHS of (14) and calculate the matrix elements up to accuracy $O\left(\sqrt{x^{2}}\right)$ which corresponds to the $O(1 / Q)$ accuracy in the DVCS amplitude. To collect contributions with such accuracy we first keep the vector $x$ off light-cone and after performing the differentiation with respect to vector $x$ we assign to the vector $x$ the value on the light-cone $x \rightarrow z, z^{2}=0$.

Let us illustrate the main steps of the calculations on the example of matrix element of the vector operator. The first term in the RHS of the (14) can easily be computed with the result:

$$
\begin{aligned}
& \left\langle p^{\prime}\left|\frac{\mathcal{D}^{\mu}}{(\mathcal{D} x)} \bar{\psi}(x) x \psi(-x)\right| p\right\rangle=-\frac{\Delta^{\mu}}{2 \xi}\left\langle\left\langle\gamma_{+}\right\rangle\right\rangle\left(T_{1}[P z, \xi]+T_{2}[P z, \xi]\right)+ \\
& +\frac{\Delta^{\mu}}{2 \xi}\left\langle\left\langle\frac{1}{M}\right\rangle\right\rangle T_{2}[P z, \xi]+\ldots
\end{aligned}
$$

where ellipses stand for the twist-4 and higher corrections. To get the above expression we used that

$$
\gamma_{\mu} z^{\mu} /(P z)=\gamma_{\mu} n^{\mu} \equiv \gamma_{+}
$$

and the Gordon identity:

$$
\left\langle\left\langle\frac{i \sigma^{\alpha \beta} x_{\alpha} \Delta_{\beta}}{2 M}\right\rangle\right\rangle=-(P x)\left\langle\left\langle\frac{1}{M}\right\rangle\right\rangle+\langle\langle x \phi\rangle,
$$

which follows from the equations of motion for nucleon Dirac spinors:

$$
(\not p-M) U(p, S)=0, \quad \bar{U}\left(p^{\prime}, S^{\prime}\right)\left(\not p^{\prime}-M\right)=0 .
$$

Consider now a more complicated matrix element of the second term in RHS of (14). Using (12), (15) and (22) we obtain for this term the following expression:

$$
\begin{aligned}
2^{\text {nd }} \text { term } & =\frac{1}{2} \int_{0}^{1} d \alpha\left\{e^{-i \bar{\alpha}(\Delta x)}+e^{i \bar{\alpha}(\Delta x)}\right\}\left[\partial_{\mu}-\frac{\Delta_{\mu}}{(\Delta x)}(x \partial)\right] \\
& \times\left(\langle\langle x\rangle\rangle\left(T_{1}[(P x), \xi(x)]+T_{2}[(P x), \xi(x)]\right)-\left\langle\left\langle\frac{(P x)}{M}\right\rangle\right\rangle T_{2}[\alpha(P x), \xi(x)]\right) .
\end{aligned}
$$

Note, we can put $x=z$ only after differentiation! The latter can be done with the help of the following formulae:

$$
\left.\partial_{\mu} T_{i}[\alpha(P x), \xi(x)]\right|_{x=z}=\frac{1}{(P z)}\left[P^{\mu} \alpha \frac{\partial}{\partial \alpha}-\frac{1}{2} \Delta_{\perp}^{\mu} \frac{\partial}{\partial \xi}\right] T_{i}[\alpha(P z), \xi],
$$




$$
\left.\left[\partial_{\mu}-\frac{\Delta_{\mu}}{(\Delta x)}(x \partial)\right] T_{i}[\alpha(P x), \xi(x)]\right|_{x=z}=\frac{1}{(P z)} \frac{\Delta_{\perp}^{\mu}}{2 \xi}\left(\alpha \frac{\partial}{\partial \alpha}-\xi \frac{\partial}{\partial \xi}\right) T_{i}[\alpha(P z), \xi] .
$$

Applying these formulae and using simple identity:

$$
\left.P^{\mu}\langle\langle\not\rangle\rangle\right\rangle=(P z)\left\langle\left\langle\gamma^{\mu}\right\rangle\right\rangle-(P z)\left\langle\left\langle\gamma_{\perp}^{\mu}\right\rangle\right\rangle,
$$

we obtain for the second term the following expression:

$$
\begin{aligned}
2^{\text {nd }} \text { term }= & \frac{1}{2} \int_{0}^{1} d \alpha\left\{e^{i \bar{\alpha} 2 \xi(P x)}+e^{-i \bar{\alpha} 2 \xi(P x)}\right\}\left(\left\langle\gamma_{\perp}^{\mu}\right\rangle\right\rangle\left(T_{1}+T_{2}\right)[\alpha(P z), \xi]+ \\
& +\frac{\Delta_{\perp}^{\mu}}{2 \xi}\left\langle\left\langle\gamma_{+}\right\rangle\right\rangle\left(1+\alpha \frac{\partial}{\partial \alpha}-\xi \frac{\partial}{\partial \xi}\right)\left(T_{1}+T_{2}\right)[\alpha(P z), \xi]- \\
& -\frac{\Delta_{\perp}^{\mu}}{2 \xi}\left\langle\left\langle\frac{1}{M}\right\rangle\right\rangle\left(1+\alpha \frac{\partial}{\partial \alpha}-\xi \frac{\partial}{\partial \xi}\right) T_{2}[\alpha(P z), \xi] .
\end{aligned}
$$

Using the definition of the amplitudes $T_{1,2}$ Eqs. (17) this term can be represented in the form which is convenient for the transition to the momentum space:

$$
2^{\text {nd }} \text { term }=\int_{-1}^{1} d u G^{\mu}(u, \xi) \int_{0}^{1} d \alpha\left\{e^{i 2(P x)[\alpha(u-\xi)+\xi]}+e^{i 2(P x)[\alpha(u+\xi)-\xi]}\right\},
$$

where

$$
\begin{aligned}
G^{\mu}(u, \xi)= & \left\langle\left\langle\gamma_{\perp}^{\mu}\right\rangle\right\rangle(H+E)(u, \xi)+\frac{\Delta_{\perp}^{\mu}}{2 \xi}\left\langle\left\langle\frac{1}{M}\right\rangle\right\rangle\left[u \frac{\partial}{\partial u}+\xi \frac{\partial}{\partial \xi}\right] E(u, \xi)- \\
& -\frac{\Delta_{\perp}^{\mu}}{2 \xi}\left\langle\left\langle\gamma_{+}\right\rangle\right\rangle\left[u \frac{\partial}{\partial u}+\xi \frac{\partial}{\partial \xi}\right](H+E)(u, \xi) .
\end{aligned}
$$

Manipulations with the nucleon matrix element of the third term of the operator identity (14) are similar. For this term we obtain:

$$
3^{\mathrm{d}} \text { term }=i \epsilon_{\mu k}^{\perp} \int_{-1}^{1} d u \widetilde{G}^{k}(u, \xi) \int_{0}^{1} d \alpha\left\{e^{i 2(P x)[\alpha(u-\xi)+\xi]}-e^{i 2(P x)[\alpha(u+\xi)-\xi]}\right\},
$$

where

$$
\begin{aligned}
\widetilde{G}^{k}(u, \xi)= & \left\langle\left\langle\gamma_{\perp}^{\mu} \gamma_{5}\right\rangle\right\rangle \widetilde{H}(u, \xi)+\frac{1}{2} \Delta_{\perp}^{\mu}\left\langle\left\langle\frac{\gamma_{5}}{M}\right\rangle\right\rangle\left[1+u \frac{\partial}{\partial u}+\xi \frac{\partial}{\partial \xi}\right] \widetilde{E}(u, \xi)- \\
& -\frac{\Delta_{\perp}^{\mu}}{2 \xi}\left\langle\left\langle\gamma_{+} \gamma_{5}\right\rangle\right\rangle\left[u \frac{\partial}{\partial u}+\xi \frac{\partial}{\partial \xi}\right] \widetilde{H}(u, \xi) .
\end{aligned}
$$

Collecting all terms (20),(29) and (31) and performing the Fourier transformation on the light-cone we obtain the expression for $F_{\mu}$ (see definition (⿴)) in the WW approximation in terms of twist-2 SPD's $H, E$ and $\widetilde{H}, \widetilde{E}$ :

$$
\begin{aligned}
F_{\mu}^{W W}(x, \xi)= & \frac{\Delta_{\mu}}{2 \xi}\left\langle\left\langle\frac{1}{M}\right\rangle\right\rangle E(x, \xi)-\frac{\Delta_{\mu}}{2 \xi}\left\langle\left\langle\gamma_{+}\right\rangle\right\rangle(H+E)(x, \xi)+ \\
& +\int_{-1}^{1} d u G_{\mu}(u, \xi) W_{+}(x, u, \xi)+i \epsilon_{\perp \mu k} \int_{-1}^{1} d u \widetilde{G}^{k}(u, \xi) W_{-}(x, u, \xi),
\end{aligned}
$$


where we introduced a convenient notation for the WW-kernels:

$$
\begin{aligned}
W_{ \pm}(x, u, \xi)= & \frac{1}{2}\left\{\theta(x>\xi) \frac{\theta(u>x)}{u-\xi}-\theta(x<\xi) \frac{\theta(u<x)}{u-\xi}\right\} \\
& \pm \frac{1}{2}\left\{\theta(x>-\xi) \frac{\theta(u>x)}{u+\xi}-\theta(x<-\xi) \frac{\theta(u<x)}{u+\xi}\right\} .
\end{aligned}
$$

The function $G^{\mu}(u, \xi)$ and $\widetilde{G}^{k}(u, \xi)$ are defined in (30) and (32).

The analogous calculation for the axial matrix elements gives:

$$
\begin{aligned}
\widetilde{F}_{\mu}^{W W}(x, \xi)= & \frac{\Delta_{\mu}}{2}\left\langle\left\langle\frac{\gamma_{5}}{M}\right\rangle\right\rangle \widetilde{E}(x, \xi)-\frac{\Delta_{\mu}}{2 \xi}\left\langle\left\langle\gamma_{+} \gamma_{5}\right\rangle\right\rangle \widetilde{H}(x, \xi)+ \\
& +\int_{-1}^{1} d u \widetilde{G}_{\mu}(u, \xi) W_{+}(x, u, \xi)+i \epsilon_{\perp \mu k} \int_{-1}^{1} d u G^{k}(u, \xi) W_{-}(x, u, \xi) .
\end{aligned}
$$

Let us note that the spinor structures in the functions $G^{\mu}(u, \xi)$ and $\widetilde{G}^{k}(u, \xi)$ are not independent. They can be related to each other with the help of equations of motion for Dirac spinors (23). However, we prefer not to reduce the number of structures to retain the symmetric form of the results (33, 35).

The generalisation of the WW relations for the non-forward case was discussed first in Ref. [20]. Our results (33,35) do not agree, however, with those of Ref. [20]. The reason for the disagreement is that in Ref. [20] instead of the complete operator identity of the type (11) only its part which survives in the forward limit has been considered. This simplification is justified in order to reproduce only the WW relations in the forward case. The WW relations obtained in Ref. [11] can be reduced to the form (33),35) obtained here.

\section{Properties of the WW kernels}

In the previous section we introduced the notion of the WW-kernels, see Eq. (34). In this section we consider the general properties of these kernels. Let us introduce the following notations for the action of the WW kernels on a function $f(u, \xi)$ :

$$
W_{ \pm} \otimes f[x, \xi] \equiv \int_{-1}^{1} d u W_{ \pm}(x, u, \xi) f(u, \xi)
$$

with $W_{ \pm}$given by Eq. (34). We shall call "WW transform" the resulting functions $W_{ \pm} \otimes$ $f[x, \xi]$ and we shall call the "WW transformation" the action of the WW kernels.

Limiting cases. We consider two limiting cases of the WW transformation: the forward limit $\xi \rightarrow 0$ and the 'meson' limit $\xi \rightarrow 1$. In the forward limit we easily obtain:

$$
\begin{aligned}
\lim _{\xi \rightarrow 0} W_{+} \otimes f[x, \xi] & =\theta(x \geq 0) \int_{x}^{1} \frac{d u}{u} f(u, \xi=0)-\theta(x \leq 0) \int_{-1}^{x} \frac{d u}{u} f(u, \xi=0), \\
\lim _{\xi \rightarrow 0} W_{-} \otimes f[x, \xi] & =0
\end{aligned}
$$

We can see that the action of the $W_{+}$in the forward limit reproduces the Wandzura-Wilczek relation for the spin structure function $g_{T}$ [21]. The term with $\theta(x \geq 0)$ corresponds to the 
quark distributions and the term with $\theta(x \leq 0)$ to the antiquark ones. The $W_{-}$kernel disappears in the forward limit, so that this kernel is 'genuine non-forward' object.

In the limit $\xi \rightarrow 1$ the skewed parton distributions have properties of meson distribution amplitudes. In this limit the WW transforms have the form (we use notation $f(u, \xi=1)=$ $\varphi(u))$ :

$$
\lim _{\xi \rightarrow 1} W_{ \pm} \otimes f[x, \xi]=\frac{1}{2}\left\{\int_{-1}^{x} \frac{d u}{1-u} \varphi(u) \pm \int_{x}^{1} \frac{d u}{1+u} \varphi(u)\right\}
$$

which corresponds to the WW relations for the meson distribution amplitudes derived in [22, 23].

We can see that WW transforms of skewed parton distributions interpolate between WW relations for parton distributions and the meson distribution amplitude. Also general form of the WW kernels (34) allows to derive WW relations for distribution amplitudes of a meson of arbitrary spin.

Mellin moments: One can easily derive the Mellin moments of the WW transform. The result has the form:

$$
\int_{-1}^{1} d x x^{N} W_{ \pm} \otimes f[x, \xi]=\frac{1}{N+1} \int_{-1}^{1} d u\left[\frac{u^{N+1}-\xi^{N+1}}{u-\xi} \pm \frac{u^{N+1}-(-\xi)^{N+1}}{u+\xi}\right] f(u, \xi) .
$$

From this simple exercise we can see important property of the WW transformation, namely, if the function $f(u, \xi)$ satisfies the polynomiality condition, i.e.:

$$
\int_{-1}^{1} d u u^{N} f(u, \xi)=\text { polynomial in } \xi \text { of the order } N+1
$$

its WW transform also satisfies the polynomiality condition. Let us consider now the special cases $N=0,1$ of the Mellin moments. These particular moments of the twist-3 SPD's (7) do not receive contribution from the 'genuine twist-3' quark-gluon operators, therefore the WW approximation for them gives exact results. In the forward limit this observation leads to Burkhard-Cottingham $(N=0)$ [24] and Efremov-Leader-Teryaev $(N=1)$ [25] sum rules for polarized structure function $g_{T}$. The corresponding generalizations of these sum rules in the case of skewed parton distributions have been discussed in Refs. [10, 16]. From general expression (38) one gets:

$$
\begin{aligned}
\int_{-1}^{1} d x W_{-} \otimes f[x, \xi] & =0 \\
\int_{-1}^{1} d x W_{+} \otimes f[x, \xi] & =\int_{-1}^{1} d u f(u, \xi), \\
\int_{-1}^{1} d x x W_{-} \otimes f[x, \xi] & =\frac{\xi}{2} \int_{-1}^{1} d u f(u, \xi), \\
\int_{-1}^{1} d x x W_{+} \otimes f[x, \xi] & =\frac{1}{2} \int_{-1}^{1} d u u f(u, \xi) .
\end{aligned}
$$

Below we shall use these results for the consistency checks of our results for skewed parton distributions and for derivation of the sum rules for the distributions $F_{\mu}$ and $\widetilde{F}_{\mu}(\bar{\nabla})$. 
Discontinuities. In Ref. [16] it was demonstrated that the twist-3 skewed parton distributions in the WW approximation exhibit discontinuities at the points $x= \pm \xi$. This feature is related to the properties of the WW kernels. Let us compute the discontinuities of a WW transform at the points $x= \pm \xi$ :

$$
\begin{aligned}
\lim _{\delta \rightarrow 0}\left[W_{ \pm} \otimes f[\xi+\delta, \xi]-W_{ \pm} \otimes f[\xi-\delta, \xi]\right] & =\frac{1}{2} v p \int_{-1}^{1} \frac{d u}{u-\xi} f(u, \xi) \\
\lim _{\delta \rightarrow 0}\left[W_{ \pm} \otimes f[-\xi+\delta, \xi]-W_{ \pm} \otimes f[-\xi-\delta, \xi]\right] & = \pm \frac{1}{2} v p \int_{-1}^{1} \frac{d u}{u+\xi} f(u, \xi)
\end{aligned}
$$

Here $v p$ means an integral in the sense of valeur principal. We see that for a very wide class of functions $f(u, \xi)$ the discontinuity of the corresponding WW transforms is nonzero. This feature of the WW transformation may lead to the violation of the factorisation for the twist-3 DVCS amplitude.

\section{Properties of the skewed distributions $F_{\mu}$ and $\widetilde{F}_{\mu}$}

In this section we analyse the general properties of the skewed parton distributions $F_{\mu}^{W W}$ and $\widetilde{F}_{\mu}^{W W}$ (7) in the WW approximation. We consider the limiting cases and derive sum rules for these SPD's which are valid beyond WW approximation.

Forward limit: In the forward limit the 'vector' SPD $F_{\mu}$ in the WW approximation (33) vanishes, because unpolarized parton densities of twist-3 are absent. The forward limit for the 'axial' SPD $\widetilde{F}_{\mu_{\perp}}^{W W}$, see (35), is nontrivial:

$$
\begin{array}{cc}
\lim _{\Delta \rightarrow 0} \widetilde{F}_{\mu_{\perp}}^{W W}(x, \xi)=2 S_{\mu}^{\perp}\left[\Delta q(x)+\int_{x}^{1} \frac{d u}{u} \Delta q(u)\right] & \text { for } x \geq 0, \\
\lim _{\Delta \rightarrow 0} \widetilde{F}_{\mu_{\perp}}^{W W}(x, \xi)=2 S_{\mu}^{\perp}\left[\Delta \bar{q}(|x|)+\int_{|x|}^{1} \frac{d u}{u} \Delta \bar{q}(u)\right] & \text { for } x \leq 0 .
\end{array}
$$

It is easy to recognize in these equations the Wandzura-Wilczek relations for $g_{T}(x)$ [21]. In the derivation (42) we used that twist-2 skewed distribution $\widetilde{H}$ is reduced in the forward limit to the polarized quark (for $x \geq 0$ ) and antiquark (for $x \leq 0$ ) densities $\Delta q(x)$ and $\Delta \bar{q}(x)$.

Mellin moments: The lowest $N=0,1$ Mellin moments of the SPD's $F_{\mu}$ and $\widetilde{F}_{\mu}$ (7) have been analysed previously in Ref. [10]. Since for these moments the contributions from the twist-3 quark-gluon operators are absent, we have to reproduce sum rules of Ref. [10] from the WW approximation for the functions $F_{\mu}$ and $\widetilde{F}_{\mu}$, see Eqs. (33),(35). This would be a powerful check of our results.

As it follows from the definitions of twist-3 SPD's (7), the $N=0$ moments are reduced to the nucleon matrix elements of the local currents which are parametrized in terms of electric, magnetic, axial and pseudoscalar form factors of the nucleon:

$$
\begin{aligned}
\int_{-1}^{1} d u F_{\mu}(u, \xi) & =\left\langle\left\langle\gamma_{\mu}\right\rangle\right\rangle F_{1}\left(\Delta^{2}\right)+\left\langle\left\langle\frac{i \sigma_{\mu \nu} \Delta^{\nu}}{2 M}\right\rangle F_{2}\left(\Delta^{2}\right),\right. \\
\int_{-1}^{1} d u \widetilde{F}_{\mu}(u, \xi) & =\left\langle\left\langle\gamma_{\mu} \gamma_{5}\right\rangle\right\rangle G_{A}\left(\Delta^{2}\right)+\frac{\Delta_{\mu}}{2 M}\left\langle\left\langle\gamma_{5}\right\rangle\right\rangle G_{P}\left(\Delta^{2}\right) .
\end{aligned}
$$


Using the properties of the WW kernels (40) one can easily see that the expressions for $F_{\mu}^{W W}$ (33) and for $\widetilde{F}_{\mu}^{W W}$ (35) satisfy the general QCD sum rules (43). This is a sensitive check of our approach.

The general QCD expression for the $N=1$ moment of the SPD's $F_{\mu}$ and $\widetilde{F}_{\mu}$ can be obtained from the WW approximation because the quark-gluon operators do not contribute to this moment. Again with help of (40) we can obtain the results for the $N=1$ moments of the transverse components of the functions $F_{\mu}^{W W}$ and $\widetilde{F}_{\mu}^{W W}$ which enter the DVCS amplitude for the longitudinally polarized virtual photon. For the function $F_{\mu_{\perp}}$ we have

$$
\begin{aligned}
\int_{-1}^{1} d u u F_{\mu_{\perp}}^{W W}(u, \xi) & =\left\langle\left\langle\gamma_{\mu}^{\perp}\right\rangle\right\rangle \frac{1}{2}\left[\int_{-1}^{1} d u u\{H(u, \xi)+E(u, \xi)\}+G_{A}\left(\Delta^{2}\right)\right] \\
& +\frac{\Delta_{\mu}^{\perp}}{4 M}\langle\langle 1\rangle\rangle \frac{\partial}{\partial \xi} \int_{-1}^{1} d u u E(u, \xi)
\end{aligned}
$$

We see that the 'transverse' $\mathrm{SPD} F_{\mu_{\perp}}$ is sensitive to the combination

$$
\int_{-1}^{1} d u u(H(u, \xi)+E(u, \xi))+G_{A}\left(\Delta^{2}\right)
$$

which is related to the spin structure of the nucleon in the forward limit:

$$
\lim _{\Delta \rightarrow 0} \int_{-1}^{1} d u u\{H(u, \xi)+E(u, \xi)\}+G_{A}\left(\Delta^{2}\right)=2 J_{q}+\Delta q,
$$

where $J_{q}$ is a fraction of the total angular momentum of the nucleon carried by quarks and $\Delta q$ is the corresponding fraction of the spin. To derive Eq. (46) we made use of Ji's sum rule [2]. Also the new angular momentum sum rule (45) coincides with that previously derived in [10] where the WW approximation has not been considered. We see that the DVCS amplitude with longitudinally polarized virtual photon is also sensitive to the spin structure of the nucleon. In principle, this part of the amplitude can be extracted from the data through the angular, spin and $Q$ dependence of the differential cross section [26, 27. This may provide us with additional possibility to probe $J_{q}$ in the nucleon.

In the case of axial transverse SPD $\widetilde{F}_{\mu_{\perp}}$ we obtain from Eq. (35) the following result for the $N=1$ moment:

$$
\begin{aligned}
\int_{-1}^{1} d u u \widetilde{F}_{\mu_{\perp}}^{W W}(u, \xi) & =\left\langle\left\langle\gamma_{\mu}^{\perp} \gamma_{5}\right\rangle\right\rangle \frac{1}{2}\left[\int_{-1}^{1} d u u \widetilde{H}(u, \xi)+\xi^{2}\left\{F_{1}\left(\Delta^{2}\right)+F_{2}\left(\Delta^{2}\right)\right\}\right] \\
& + \text { other structures }
\end{aligned}
$$

For simplicity we do not write all possible spinor structures which appear in the RHS (47). For the comparison with the sum rules of Ref. [10] we need only coefficient in front of structure $\left\langle\left\langle\gamma_{\perp}^{\mu} \gamma_{5}\right\rangle\right\rangle$. We obtained that our result (47) is in agreement with Ref. [10]. In the forward limit we reproduce from the Eq. (47) the Efremov-Leader-Teryaev sum rule 25] for the polarized structure function $g_{T}(x)$.

Discontinuities: Using general properties of the WW transformation (41) and Eqs. (33) and (35) one obtains that $F_{\mu}^{W W}$ and $\widetilde{F}_{\mu}^{W W}$ have discontinuities at the points $x= \pm \xi$. But using a certain symmetry of the equations (33) and (35), one can find that some combinations of the 
distributions $F_{\mu}^{W W}$ and $\widetilde{F}_{\mu}^{W W}$ are free of discontinuities. For example, using formulae (41) one can see that the combination:

$$
F_{\mu}^{W W}(x, \xi)-i \varepsilon_{\perp \mu \rho} \widetilde{F}_{\rho}^{W W}(x, \xi)
$$

has no discontinuity at $x=\xi$. On the other hand, the 'dual' combination:

$$
F_{\mu}^{W W}(x, \xi)+i \varepsilon_{\perp \mu \rho} \widetilde{F}_{\rho}^{W W}(x, \xi),
$$

is free of the discontinuity at $x=-\xi$. As we shall see below, the cancellation of discontinuities in these particular combinations of the SPD's ensures the factorisation of the twist-3 DVCS amplitude on the nucleon.

\section{Pion pole and D-term in $F_{\mu}$ and $\widetilde{F}_{\mu}$}

In the Wandzura-Wilczek approximation the new skewed distributions $F_{\mu}$ and $\widetilde{F}_{\mu}$ (see Eq. (7) for definition) are fixed completely in terms of the twist-2 SPD's $H, E$ and $\widetilde{H}, \widetilde{E}$. These functions have some specific contributions which are invisible in the forward limit. In this section we consider how these special contributions are incorporated into the structure of the twist-3 distributions.

Pion pole contribution: The skewed parton distribution $\widetilde{E}$ at small $t$ is dominated by chiral contribution of the pion pole [28, 29, 30, 31] of the form:

$$
\widetilde{E}^{\text {pion pole }}(x, \xi)=\frac{4 g_{A}^{2} M^{2}}{-t+m_{\pi}^{2}} \frac{1}{\xi} \varphi_{\pi}\left(\frac{x}{\xi}\right) \theta(|x| \leq \xi),
$$

where $g_{A}$ is the axial charge of the nucleon and $\varphi_{\pi}(u)$ is the pion distribution amplitude. In the WW relation (33) the twist-2 SPD $\widetilde{E}$ enters only in the combination:

$$
\left[1+u \frac{\partial}{\partial u}+\xi \frac{\partial}{\partial \xi}\right] \widetilde{E}(u, \xi)
$$

One can easily see that the contribution of the pion pole (50) nullifies under action of the differential operator in Eq. (51). The only place where the pion pole contribution survives is the SPD $\widetilde{F}_{\mu}$, see Eq. (35). In this way we come to the following simple results for the contribution of the pion pole to the twist-3 SPD's in the WW approximation:

$$
\begin{aligned}
F_{\mu}^{W W, \text { pion pole }}(x, \xi) & =0 \\
\widetilde{F}_{\mu}^{W W, \text { pion pole }}(x, \xi) & =\frac{\Delta_{\mu}}{2 M}\left\langle\left\langle\gamma_{5}\right\rangle\right\rangle \frac{4 g_{A}^{2} M^{2}}{-t+m_{\pi}^{2}} \frac{1}{\xi} \varphi_{\pi}\left(\frac{x}{\xi}\right) \theta(|x| \leq \xi) .
\end{aligned}
$$

We see an interesting result that the pion pole contribution to the twist-3 SPD's is expressed in terms of twist-2 pion distributions amplitude. This observation is in nice agreement with the fact that in the WW approximation there exists no twist-3 pion distribution amplitude associated with vector- and axial-vector operators [32, 33. 
D-term contribution: Now we discuss a specific contribution to the twist-2 SPD's $H$ and $E$ which was found in Ref. [34]. This contribution completes the parametrisation of SPD's in terms of double distributions [35]. This contribution has the form:

$$
\begin{aligned}
& H^{\mathrm{D}-\operatorname{term}}(x, \xi)=D\left(\frac{x}{\xi}\right) \theta(|x| \leq \xi), \\
& E^{\mathrm{D}-\operatorname{term}}(x, \xi)=-D\left(\frac{x}{\xi}\right) \theta(|x| \leq \xi),
\end{aligned}
$$

where the function $D(u)$ is odd in its argument. The specifics of the D-term contribution is that it is not 'generated' by double distributions. Numerically it can be rather large as it follows from estimates in the chiral quark-soliton model of the nucleon [36]. The importance of the D-term is especially emphasized by the fact that it contributes considerably to the DVCS amplitude but drops out in Ji's angular momentum sum rule [2] because in the combination $H+E$ D-term cancels. Now if we substitue the D-term contributions (53) to our WW relations (33, 35) we see that the result is:

$$
\begin{aligned}
F_{\mu}^{\mathrm{WW} \text { D-term }}(x, \xi) & =-\frac{\Delta_{\mu}}{2 \xi M}\langle\langle 1\rangle\rangle\left(\frac{x}{\xi}\right) \theta(|x| \leq \xi), \\
\widetilde{F}_{\mu}^{\mathrm{WW} \mathrm{D}-\operatorname{term}}(x, \xi) & =0 .
\end{aligned}
$$

Again we reproduced the result anticipated in Ref. [10].

\section{DVCS amplitude}

Here we analyse the general structure of the DVCS amplitude on the nucleon to the twist-3 accuracy. To this accuracy the amplitude receives contributions not only from the transverse polarization of the virtual photon (as it is at the twist-2 level), but also from the longitudinal one.

The amplitude with the transverse polarization of the virtual photon has the form Eq. (6))

$$
\begin{aligned}
e_{\mu}^{\perp}(q) T^{\mu \nu} & =\frac{1}{2} \int_{-1}^{1} d x e_{\mu}^{\perp}(q)\left\{\left[\left(-g^{\mu \nu}\right)_{\perp}-\frac{P^{\nu} \Delta_{\perp}^{\mu}}{(P q)}\right] F_{+}(x, \xi) C^{+}(x, \xi)\right. \\
& \left.-\left[\left(-g^{\nu k}\right)_{\perp}-\frac{P^{\nu} \Delta_{\perp}^{k}}{(P q)}\right] i \epsilon_{k \mu}^{\perp} \widetilde{F}_{+}(x, \xi) C^{-}(x, \xi)\right\} .
\end{aligned}
$$

We see that in this case the amplitude to the $1 / Q$ accuracy is fixed completely in terms of the same combinations of the twist-2 SPD's. Such result for the DVCS amplitude to the $1 / Q$ accuracy confirms the ad hoc prescription suggested in Ref. [17, 18]. However the twist-3 DVCS amplitude also receives contribution from the longitudinal polarization of the virtual photon (see Eq. (6)):

${ }^{* *}$ We neglected the last term in the expression (6) for the amplitude as it being contracted with polarization of the final real photon contributes only to the order $O\left(1 / Q^{2}\right)$ which is beyond our accuracy 


$$
\begin{aligned}
e_{\mu}^{L}(q) T^{\mu \nu} & \propto\left[\left(-g^{\nu k}\right)_{\perp}-\frac{P^{\nu} \Delta_{\perp}^{k}}{(P q)}\right] \\
& \times \int_{-1}^{1} d x\left\{F_{k}(x, \xi) C^{+}(x, \xi)-i \epsilon_{\perp k \rho} \widetilde{F}_{\rho}(x, \xi) C^{-}(x, \xi)\right\} .
\end{aligned}
$$

This part of the amplitude depends on the 'transverse' parts of SPD's $F_{\mu_{\perp}}$ and $\widetilde{F}_{\mu_{\perp}}$ which are related to twist-2 SPD's $H, E$ and $\widetilde{H}, \widetilde{E}$ only in Wandzura-Wilczek approximation. Since the coefficient functions $C^{ \pm}(x, \xi)$ have singularities at points $x= \pm \xi$ the integral over $x$ may diverge due to discontinuities of SPD's in WW approximation. However it is easy to see that near the point $x=\xi$ SPD's enter in the combination (48) and near the point $x=-\xi$ in the combination (49). These are exactly the combinations which are free of discontinuities at the corresponding point, therefore the amplitude (56) is well defined in the WW approximation. The same cancellation of the discontinuities was observed in the case of DVCS on the pion target [12, 16]. In Ref. [16] some general arguments why this phenomenon should persist to the case of DVCS on any target was given. Here we have checked by the explicit calculations this conjecture for the case of the nucleon target.

\section{Conclusions}

We have presented a simple and detailed derivation of the Wandzura-Wilczek relations for the twist-3 skewed parton distributions in the nucleon. These relations allow to express the DVCS amplitude on the nucleon to the twist-3 accuracy $(1 / Q)$ in terms of the twist-2 SPD's $H, E$ and $\widetilde{H}, \widetilde{E}$.

The Wandzura-Wilczek approximation corresponds to neglecting the nucleon matrix elements of the twist-3 quark-gluon operators. It would be very interesting to check in various models of the nucleon structure whether the neglected matrix elements are indeed small. The theory of instanton vacuum predicts that in the case of polarized structure functions $g_{T}(x)$ and $h_{L}$ the corresponding matrix elements are parametrically small in the packing fraction of the instantons in the QCD vacuum, for details see [14, 15]. Another interesting possibility to check the hypothesis behind the WW approximation is the model where SPD's are represented as an overlap of nucleon wave functions [37]. The quark-gluon correlations appear in this case as the overlap between the lowest Fock component of the nucleon wave function and that with an additional gluon.

We have checked by an explicit calculation that although the SPD's in the WW approximation have discontinuities, the corresponding would-be divergences in the twist-3 DVCS amplitude are cancelled exactly and do not lead to problems with the factorization. Of course, this is not a proof of the factorization for the twist-3 DVCS amplitude.

We rederive the new angular momentum sum rule of Ref. [10] which allows us to relate twist-3 SPD's to the angular momentum fraction carried by quarks in the nucleon. Note that this sum rule is valid beyond the WW approximation. Since, in principle, the twist-3 SPD's can be extracted from the experimental data by considering $Q$, spin and angular dependencies

of the DVCS observables, this opens a complementary approach to the measurement of the quark angular momentum distribution, compared to the original proposal by Ji [2]. 
The twist-3 contributions are suppressed in the DVCS amplitude relative to the twist2 one by one power of $1 / Q$ only. Therefore, estimates of these corrections are extremely important for the possibility to extract twist-2 SPD's from experimental data. The size of the twist-3 corrections increases with the momentum transfer squared $t$, because these corrections typically enter the amplitude as $\sqrt{-t} / Q$. Their studies are hence, also helpful for the analysis of the $t$-dependence of twist-2 SPD. The latter task is mandatory for the very possibility to extract information about $L_{q}$ from the data because $L_{q}$ is related to the SPD's only at $t=0$. This point lies outside the physical region of the DVCS process so that extrapolation is required.

\section{Acknowledgments.}

We would like to thank I. Anikin, V. Braun, A. Belitsky, L. Frankfurt, K. Goeke, L. Mankiewicz, D. Müller, M. Penttinen, P. Pobylitsa, A. Radyushkin, A. Schäfer, M. Strikman, M. Vanderhaeghen, and C. Weiss for fruitful discussions. M.V.P. acknowledges kind hospitality of BNL and Penn State University where a part of this work has been done. The work of N.K. was supported by the DFG, project No. 920585. M.V.P. is supported by DFG, BMFB and COSY.

\section{APPENDIX:}

For the convenience of the reader who may wish to use our results for the calculation of DVCS observables to the twist-3 accuracy, we collect the resulting expressions for the DVCS amplitude to the accuracy $O(1 / Q)$ in the Wandzura-Wilczek approximation. We present the results in the form which can be easily used for the analysis of DVCS observables.

The DVCS amplitude on the nucleon to the order $O(1 / Q)$ obtained in Refs. [10, 11] has the form:

$$
\begin{aligned}
T^{\mu \nu} & =\frac{1}{2} \int_{-1}^{1} d x\left\{\left[\left(-g^{\mu \nu}\right)_{\perp}-\frac{P^{\nu} \Delta_{\perp}^{\mu}}{(P q)}\right] n^{\beta} F_{\beta}(x, \xi) C^{+}(x, \xi)\right. \\
& -\left[\left(-g^{\nu k}\right)_{\perp}-\frac{P^{\nu} \Delta_{\perp}^{k}}{(P q)}\right] i \epsilon_{k \mu}^{\perp} n^{\beta} \widetilde{F}_{\beta}(x, \xi) C^{-}(x, \xi) \\
& \left.-\frac{(q+4 \xi P)^{\mu}}{(P q)}\left[\left(-g^{\nu k}\right)_{\perp}-\frac{P^{\nu} \Delta_{\perp}^{k}}{(P q)}\right]\left\{F_{k}(x, \xi) C^{+}(x, \xi)-i \epsilon_{k \rho}^{\perp} \widetilde{F}_{\rho}(x, \xi) C^{-}(x, \xi)\right\}\right\}
\end{aligned}
$$

where to the twist-3 accuracy:

$$
\begin{aligned}
& P=\frac{1}{2}\left(p+p^{\prime}\right)=n^{*}, \quad \Delta=p^{\prime}-p=-2 \xi P+\Delta_{\perp}, \\
& q=-2 \xi P+\frac{Q^{2}}{4 \xi} n, \quad q^{\prime}=q-\Delta=\frac{Q^{2}}{4 \xi} n-\Delta_{\perp}
\end{aligned}
$$

where $p, p^{\prime}$ are momenta of the initial and final nucleon and $q, q^{\prime}$ are momenta of the initial and final photon respectively. The light-like vectors $n$ and $n^{*}$ are normalized as $\left(n \cdot n^{*}\right)=1$. 
Also we introduce the metric and totally antisymmetric tensors in the two dimensional transverse plane $\left(\varepsilon_{0123}=+1\right)$ :

$$
\left(-g^{\mu \nu}\right)_{\perp}=-g^{\mu \nu}+n^{\mu} n^{* \nu}+n^{\nu} n^{* \mu}, \quad \epsilon_{\mu \nu}^{\perp}=\epsilon_{\mu \nu \alpha \beta} n^{\alpha} P^{\beta}
$$

The leading order coefficient functions are:

$$
C^{ \pm}(x, \xi)=\frac{1}{x-\xi+i \varepsilon} \pm \frac{1}{x+\xi-i \varepsilon} .
$$

The skewed parton distributions $F_{\mu}$ and $\widetilde{F}_{\mu}$ can be related to the twist-2 SPD's $H, E, \widetilde{H}$ and $\widetilde{E}$ with help of Wandzura-Wilczek relations:

$$
\begin{aligned}
F_{\mu}^{W W}(x, \xi)= & \frac{\Delta_{\mu}}{2 \xi}\left\langle\left\langle\frac{1}{M}\right\rangle\right\rangle E(x, \xi)-\frac{\Delta_{\mu}}{2 \xi}\left\langle\left\langle\gamma_{+}\right\rangle\right\rangle(H+E)(x, \xi)+ \\
& +\int_{-1}^{1} d u G_{\mu}(u, \xi) W_{+}(x, u, \xi)+i \epsilon_{\perp \mu k} \int_{-1}^{1} d u \widetilde{G}^{k}(u, \xi) W_{-}(x, u, \xi), \\
\widetilde{F}_{\mu}^{W W}(x, \xi)= & \Delta_{\mu} \frac{1}{2}\left\langle\left\langle\frac{\gamma_{5}}{M}\right\rangle \widetilde{E}(x, \xi)-\frac{\Delta_{\mu}}{2 \xi}\left\langle\left\langle\gamma_{+} \gamma_{5}\right\rangle\right\rangle \widetilde{H}(x, \xi)+\right. \\
& +\int_{-1}^{1} d u \widetilde{G}_{\mu}(u, \xi) W_{+}(x, u, \xi)+i \epsilon_{\perp \mu k} \int_{-1}^{1} d u G^{k}(u, \xi) W_{-}(x, u, \xi)
\end{aligned}
$$

The following notations are used:

$$
\begin{aligned}
& G^{\mu}(u, \xi)=\left\langle\left\langle\gamma_{\perp}^{\mu}\right\rangle\right\rangle(H+E)(u, \xi)+\frac{\Delta_{\perp}^{\mu}}{2 \xi}\left\langle\left\langle\frac{1}{M}\right\rangle\left[u \frac{\partial}{\partial u}+\xi \frac{\partial}{\partial \xi}\right] E(u, \xi)-\right. \\
&-\frac{\Delta_{\perp}^{\mu}}{2 \xi}\left\langle\left\langle\gamma_{+}\right\rangle\right\rangle\left[u \frac{\partial}{\partial u}+\xi \frac{\partial}{\partial \xi}\right](H+E)(u, \xi) \\
& \widetilde{G}^{\mu}(u, \xi)=\left\langle\left\langle\gamma_{\perp}^{\mu} \gamma_{5}\right\rangle\right\rangle \widetilde{H}(u, \xi)+\frac{1}{2} \Delta_{\perp}^{\mu}\left\langle\left\langle\frac{\gamma_{5}}{M}\right\rangle\right\rangle\left[1+u \frac{\partial}{\partial u}+\xi \frac{\partial}{\partial \xi}\right] \widetilde{E}(u, \xi)- \\
&- \frac{\Delta_{\perp}^{\mu}}{2 \xi}\left\langle\left\langle\gamma_{+} \gamma_{5}\right\rangle\right\rangle\left[u \frac{\partial}{\partial u}+\xi \frac{\partial}{\partial \xi}\right] \widetilde{H}(u, \xi) \\
& W_{ \pm}(x, u, \xi) \quad=\frac{1}{2}\left\{\theta(x>\xi) \frac{\theta(u>x)}{u-\xi}-\theta(x<\xi) \frac{\theta(u<x)}{u-\xi}\right\} \\
& \pm \frac{1}{2}\left\{\theta(x>-\xi) \frac{\theta(u>x)}{u+\xi}-\theta(x<-\xi) \frac{\theta(u<x)}{u+\xi}\right\} .
\end{aligned}
$$

The sandwiching between nucleon Dirac spinors is denoted by $\langle\langle\ldots\rangle\rangle=\bar{U}\left(p^{\prime}\right) \ldots U(p)$ and the quarks flavour dependence in the amplitude can be easily restored by the substitution:

$$
F_{\mu}\left(\widetilde{F}_{\mu}\right) \rightarrow \sum_{f=u, d, s, \ldots} e_{f}^{2} F_{\mu}^{f}\left(\widetilde{F}_{\mu}^{f}\right) .
$$




\section{References}

[1] D. Müller, D. Robaschik, B. Geyer, F.M. Dittes, J. Horejsi, Fortschr. Phys. 42 (1994) 101.

[2] X. Ji, Phys. Rev.D55 (1997) 7114.

[3] P. R. Saull [ZEUS Collaboration], "Prompt photon production and observation of deeply virtual Compton scattering," hep-ex/0003030.

[4] Rainer Stamen [H1-Collaboration], “ Measurement of the Deeply Virtual Compton Scattering at Hera", H1prelim-00-17, DIS 2000 and IHEP 2000.

[5] M. Amarian [HERMES collaboration], "DVCS and exclusive meson production measured by HERMES", talk at workshop "Skewed Parton Distributions and Lepton - Nucleon Scattering", DESY, Sept. 2000, http://hermes.desy.de/workshop/TALKS/talks.htm]

[6] A. V. Radyushkin, Phys. Rev. D56 (1997), 5524.

[7] X. Ji and J. Osborne, Phys. Rev. D58 (1998) 094018.

[8] J. C. Collins and A. Freund, Phys. Rev. D59 (1999) 074009.

[9] I. V. Anikin, B. Pire and O. V. Teryaev, Phys. Rev. D62 (2000) 071501 hep$\mathrm{ph} / 0003203$.

[10] M. Penttinen, M. V. Polyakov, A. G. Shuvaev and M. Strikman, Phys. Lett. B491 (2000) 96 hep-ph/0006321.

[11] A. V. Belitsky and D. Muller, Nucl. Phys. B589 (2000) 611 hep-ph/0007031.

[12] A. V. Radyushkin and C. Weiss, Phys. Lett. B493 (2000) 332 hep-ph/0008214;

A. V. Radyushkin and C. Weiss, "DVCS amplitude at tree level: Transversality, twist-3, and factorization," hep-ph/0010296.

[13] G. S. Mitchell [E155 Collaboration], "Spin structure functions $g_{1}$ and $g_{2}$ for the proton and deuteron," hep-ex/9903055;

P. Bosted [E155x Collaboration], Nucl. Phys. A663 (2000) 297.

[14] J. Balla, M. V. Polyakov and C. Weiss, Nucl. Phys. B510 (1998) 327 [hep-ph/9707515.

[15] B. Dressler and M. V. Polyakov, Phys. Rev. D61 (2000) 097501 hep-ph/9912376.

[16] N. Kivel, M. V. Polyakov, A. Schafer and O. V. Teryaev, Phys. Lett. B497 (2001) 73 [hep-ph/0007315.

[17] P.A.M. Guichon and M. Vanderhaeghen, Prog. Part. Nucl. Phys. 41 (1998) 125.

[18] M. Vanderhaeghen, P.A.M. Guichon, M. Guidal Phys. Rev. Lett. 80 (1998) 5064. 
[19] I. I. Balitsky and V. M. Braun, Nucl. Phys. B311 (1989) 541.

[20] J. Blumlein and D. Robaschik, Nucl. Phys. B581 (2000) 449 hep-ph/0002071.

[21] S. Wandzura and F. Wilczek, Phys. Lett. B72 (1977) 195.

[22] P. Ball and V. M. Braun, Phys. Rev. D54 (1996) 2182.

[23] P. Ball, V. M. Braun, Y. Koike and K. Tanaka, Nucl. Phys. B529 (1998) 323.

[24] H. Burkhardt and W. N. Cottingham, Annals Phys. 56 (1970) 453.

[25] A.V. Efremov, O.V. Teryaev, E. Leader, Phys. Rev. D55 (1997) 4307;

O.V. Teryaev, Proceedings of 11th International Symposium on High-energy Spin Physics (SPIN 94), Bloomington, Indiana, 15-22 Sep 1994, p. 467-471.

[26] M. Diehl, T. Gousset, B. Pire and J. P. Ralston, Phys. Lett. B411 (1997) 193 hepph/9706344.

[27] A. V. Belitsky, D. Muller, L. Niedermeier and A. Schafer, "Leading twist asymmetries in deeply virtual Compton scattering," hep-ph/0004059.

[28] L. L. Frankfurt, M. V. Polyakov and M. Strikman, hep-ph/9808449.

[29] L. Mankiewicz, G. Piller and A. Radyushkin, Eur. Phys. J. C10 (1999) 307 hepph/9812467]

[30] L. L. Frankfurt, P. V. Pobylitsa, M. V. Polyakov and M. Strikman, Phys. Rev. D60 (1999) 014010 hep-ph/9901429

[31] M. Penttinen, M. V. Polyakov and K. Goeke, Phys. Rev. D62 (2000) 014024 hepph/9909489].

[32] V. M. Braun and I. E. Filyanov, Z. Phys. C48 (1990) 239.

[33] P. Ball, JHEP 9901 (1999) 010 hep-ph/9812375.

[34] M.V. Polyakov and C. Weiss, Phys. Rev. D60 (1999) 114017.

[35] A. V. Radyushkin, Phys. Rev. D59 (1999) 014030 hep-ph/9805342.

[36] V. Y. Petrov, P. V. Pobylitsa, M. V. Polyakov, I. Bornig, K. Goeke and C. Weiss, Phys. Rev. D57 (1998) 4325 hep-ph/9710270.

[37] M. Diehl, T. Feldmann, R. Jakob and P. Kroll, "The Overlap Representation of Skewed Quark and Gluon Distributions," hep-ph/0009255;

S. J. Brodsky, M. Diehl and D. S. Hwang, "Light-cone wavefunction representation of deeply virtual Compton scattering," hep-ph/0009254. 